\title{
Topical capsaicin application causes cold hypersensitivity in awake monkeys
}

\author{
Hiroshi Kamo ${ }^{1)}$, Kuniya Honda ${ }^{1)}$, Junichi Kitagawa ${ }^{2,6)}$, Yoshiyuki Tsuboi ${ }^{2,6)}$, \\ Masahiro Kondo ${ }^{2,6)}$, Masato Taira ${ }^{3)}$, Akiko Yamashita ${ }^{4)}$, Narumi Katsuyama ${ }^{4)}$, \\ Yuji Masuda ${ }^{5)}$, Takafumi Kato ${ }^{5)}$ and Koichi Iwata ${ }^{2,4,6)}$ \\ ${ }^{1)}$ Department of Oral and Maxillofacial Surgery, Nihon University School of Dentistry, Tokyo, Japan \\ ${ }^{2}$ Department of Physiology, Nihon University School of Dentistry, Tokyo, Japan \\ ${ }^{3)}$ Advanced Research Institute for the Sciences and Humanities Life Science, Nihon University Advanced \\ Research Institute for the Sciences and Humanities, Tokyo, Japan \\ ${ }^{4)}$ Division of Applied System Neuroscience Advanced Medical Research Center, \\ Nihon University Graduate School of Medical Science, Tokyo, Japan \\ ${ }^{5)}$ Institute for Oral Science, Division of Oral and Maxillofacial Biology, Matsumoto Dental University, \\ Shiojiri, Japan \\ ${ }^{6}$ Division of Functional Morphology, Dental Research Center, Nihon University School of Dentistry, \\ Tokyo, Japan
}

(Received 11 March and accepted 8 April 2008)

\begin{abstract}
Recent animal studies have demonstrated that many trigeminal ganglion neurons co-express TRPV1 and TRPA1 receptors following peripheral inflammation. In the present study, we examined whether cold receptors were sensitized by capsaicin in awake monkeys. Two monkeys were trained to detect a change in cold stimulus temperature $\left(30^{\circ} \mathrm{C}\right.$ to $0.5,1.0$, 1.5 or $2.0^{\circ} \mathrm{C}$ ) applied to the facial skin. A total of 589 trials were studied, and the number of escape and hold-through trials and detection latency were measured. The number of escape trials was increased after capsaicin treatment, whereas that of hold-through trials was decreased. Detection latency was significantly decreased after capsaicin treatment. The present findings suggest that topical application of capsaicin to the facial skin induces reversible hypersensitivity to a facial cold stimulus in behaving monkeys. (J. Oral Sci. 50, 175-179, 2008)
\end{abstract}

Correspondence to Dr. Koichi Iwata, Department of Physiology, Nihon University School of Dentistry, 1-8-13 Kanda-Surugadai, Chiyoda-ku, Tokyo 101-8310, Japan

Tel: +81-3-3219-8112

Fax: +81-3-3219-8341

E-mail: iwata-k@dent.nihon-u.ac.jp
Keywords: allodynia; capsaicin; cold hyperalgesia; temperature sense; monkey.

\section{Introduction}

It has been reported that administration of capsaicin induces hyperexcitability of C- and/or A $\delta$-fibers $(1,2)$. Capsaicin is known to specifically excite $\mathrm{C}$ - and smalldiameter $\mathrm{A} \delta$ - fibers in the periphery (2). Capsaicin binds to vanilloid receptors such as TRPV1, thus generating receptor potentials, resulting in action potentials. It has also been reported that topical application of capsaicin to the skin produces reversible sensitization of $\mathrm{C}$ - and smalldiameter $A \delta$ - fibers in humans (2). Therefore, capsaicin treatment of the skin is considered to be a good procedure for studying the underlying mechanisms of peripheral sensitization without the need for any tissue damage.

A study using monkeys has also shown that topical application of capsaicin to the facial skin causes dermal flare formation and increases skin heat sensitivity (3). Recently, it has been shown that a high proportion of trigeminal ganglion (TG) neurons express TRPV1 and TRPA1 receptors, and that some also express TRPM8 receptors (4). These data suggest that TRP receptor 
sensitization sensitizes cold receptors as well as heat receptors, as TG neurons co-express both. However, it is still unclear whether capsaicin sensitizes cold receptors.

Therefore, we examined whether topical application of capsaicin to the face in behaving monkeys would increase cold sensation, and also how sensitized TRPV1 receptors are involved in cold sensitization, using a behavioral approach.

\section{Materials and Methods}

This study was approved by the Animal Experimentation Committee of Nihon University School of Dentistry. All surgery and animal care were conducted in accordance with the National Institutes of Health Guide for the Care and Use of Laboratory Animals and the Guidelines for Institutional Animal Care, and the Guidelines of the International Association for the Study of Pain (5).

\section{Animal preparation}

Two 5-year-old Japanese macaque monkeys (Macaca fuscata) weighing 5.4 and $6.2 \mathrm{~kg}$ were used for the present study.
The monkeys were anesthetized initially with ketamine hydrochloride $(10 \mathrm{mg} / \mathrm{kg}$, i.m.). Anesthesia was subsequently maintained with a mixture of halothane $(2-3 \%)$, nitrous oxide $(60 \%)$ and oxygen. The monkeys were then placed in a stereotaxic frame. A head holder for chronic experiments was implanted on the head and stabilized using screws into the skull. During the surgery, body temperature was maintained at $37-38^{\circ} \mathrm{C}$ with a heating pad, and the heart rate was continually monitored by ECG recording. Expired $\mathrm{CO}_{2}$ concentration was also monitored and maintained at a level of 3.0-4.0\%.

After the surgical procedure, the monkeys were routinely sedated with a small amount of ketamine $(2-3 \mathrm{mg} / \mathrm{kg}$, i.m.) and given penicillin (10,000 units/kg i.m.) and glucose-saline $(40-50 \mathrm{ml}$ of a $5 \%$ glucose solution in $0.18 \% \mathrm{NaCl}$, s.c.). In addition, the analgesic ketoprofen $(5 \mathrm{mg} / \mathrm{kg}$, i.m.) was administered daily after surgery for 3-4 days. Thereafter, the monkeys were tested daily until their ability to discriminate the change in intensity of the light illumination had been re-established. Then, the monkeys were trained for 2-3 months to perform two different types of detection task.
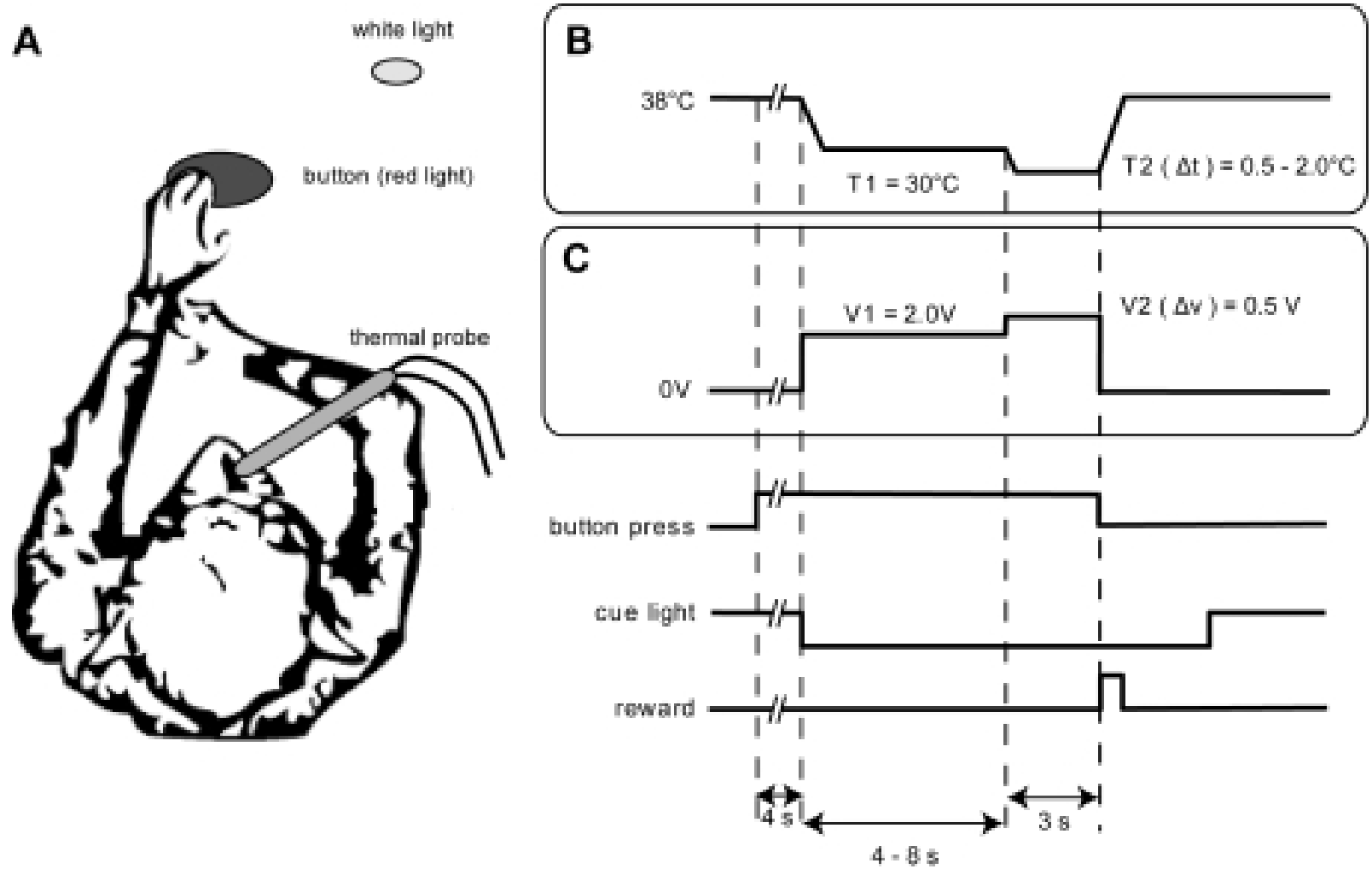

Fig. 1 Schematic illustration of the task for detecting changes in temperature shift and light illumination. The thermal probe was placed on the right side of the facial skin. The response button with a red light and white light for the light detection task was presented on the front of the panel (A). Two different detection tasks were used in the present study: cold (B) and light detection tasks (C). When the monkey pressed the button, cold or light was presented. From between 4 to 8 seconds after the initial button press, monkeys had to detect (indicated by a button release) the change in intensity of temperature or light illumination in order to obtain a reward. 


\section{Behavioral tasks}

Before the start of training, water intake was restricted to $300 \mathrm{ml}$ per day for 1 week. The training took place daily until a criterion performance had been attained, at which time initial surgery was performed. A schematic illustration of the behavioral tasks used in the present study is shown in Fig.1. The behavioral task introduced in the present study had been previously developed and refined. A thermal probe was placed on the right facial skin, and the monkeys were seated quietly in a monkey chair for 2-3 $\mathrm{h}$ (Fig. 1A). A red light was used as a signal to the monkey to press a button in order to initiate a trial. When the monkey pressed the button, three different types of tasks were presented randomly. The first task was the "cold detection task" (Fig. 1B): The monkey pressed the button, and a cold stimulus $\left(\mathrm{T} 1: 30^{\circ} \mathrm{C}\right)$ was presented to the facial skin. After a random period ranging from 4 to $8 \mathrm{~s}$, the stimulus intensity $\left(\mathrm{T} 2: 0.5^{\circ} \mathrm{C}, 1.0^{\circ} \mathrm{C}, 1.5^{\circ} \mathrm{C}\right.$ or $\left.2.0^{\circ} \mathrm{C}\right)$ decreased from the baseline stimulation level (T1). When monkeys detected the presentation of the T2 stimulus temperature within 3 $\mathrm{s}$, they received $0.5 \mathrm{ml}$ of orange juice as a reinforcer. The second task was the "light detection task" (Fig.1C): The monkey pressed the button and an illuminated light (V1: $2.0 \mathrm{~V}$ indicated as the voltage applied to the bulb) was presented in the front of the panel. After a random period ranging from 4 to $8 \mathrm{~s}$, the visual stimulus intensity increased (V2: $0.5 \mathrm{~V}$ ) above the baseline stimulation.

When monkeys showed more than $60 \%$ correct responses in the cold detection task, capsaicin was administrated to the facial skin. Four-millimolar capsaicin $(0.1 \mathrm{ml})$ was soaked into a cotton patch $(8 \times 8 \mathrm{~mm})$ and placed on the facial skin. One hour after capsaicin treatment, the patch was removed and the thermal probe was replaced on the same facial skin. Then the stimulus detection session was run. The percentage of correct trials, percentage holdthrough trials in which the monkeys did not respond to the change in temperature (T2) or light (V2) intensity change during trials, percentage escape trials, and $\mathrm{T} 2$ or V2 detection latency were studied.

In all the tasks where cold was presented, the monkeys were able to stop the cold stimulation at any time by releasing the button (escape trial). However, if the release behavior came before the presentation of the $\mathrm{T} 2$ or V2 stimulus presentation, no reward was given for that trial.

\section{Results}

The change in nocifensive behavior following cold stimulation of the face was studied in capsaicin-treated monkeys. A total of 414 cold and 175 light detection trials were studied in the behaving monkeys.

\section{Change in correct, hold-through and escape performance}

Before capsaicin treatment, monkeys performed the cold detection task correctly in $63 \%$ of the trials (Fig. 2A). The percentage of correct trials increased to $71 \%$ after capsaicin treatment (Fig. 2B). On the other hand, the percentage of escapes increased from $18 \%$ to $21 \%$ after capsaicin treatment (Fig. 2A and B). We also calculated the percentage of hold-through trials. A hold-through trial was defined when monkeys were unable to detect the small change in temperature or light. Therefore, the holdthrough trials were considered trials where the monkeys failed to detect the T2 or V2 change. We observed a decrease in hold-through trials after capsaicin treatment (19\% to $8 \%$ ) (Fig. 2A and B). The percentage of correct trials was very high in light detection trials under normal conditions (before capsaicin, $98 \%$; after capsaicin, $90 \%$ ) as illustrated in Fig. 2C and D. Escape (before capsaicin, $1 \%$; after capsaicin, $8 \%$ ) and hold-though (before capsaicin, $1 \%$; after capsaicin, $2 \%$ ) trials increased after capsaicin treatment (Fig. 2C and D).

\section{Change in detection latency after capsaicin treatment}

The frequency histograms of heat detection latencies are presented in Fig. 3. Mean cold detection latencies were
A

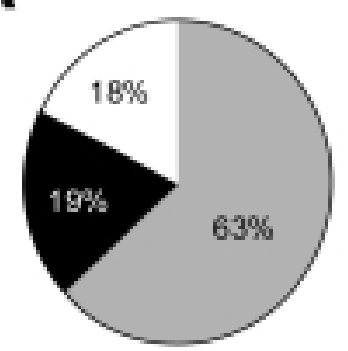

C

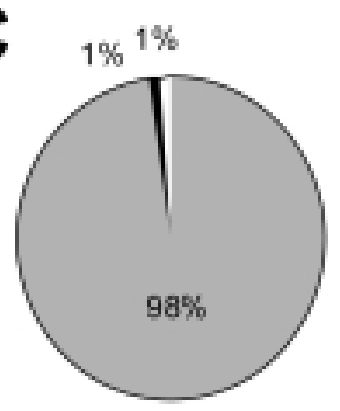

B

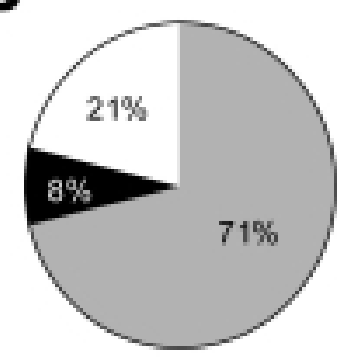

D

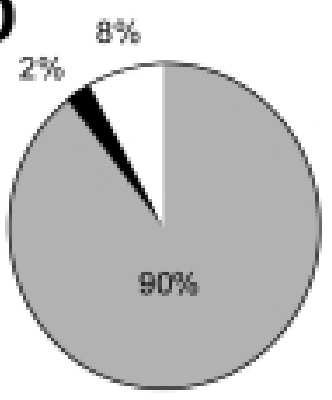

\section{Correct}

: Hold - through Escape

Fig. 2 Percentage correct, escape and hold-through trials during cold and light detection trials. Data are calculated from 589 cold and light detection trials. 
calculated from 414 trials. The cold detection latency differed at different $\mathrm{T} 2$ temperatures. Detection latencies were significantly shorter when the $\mathrm{T} 2$ temperature shift was smaller (before capsaicin treatment: $\mathrm{T} 2=0.5^{\circ} \mathrm{C}, 1.6$ $\pm 0.2 \mathrm{~s} ; \mathrm{T} 2=1.0^{\circ} \mathrm{C}, 1.4 \pm 0.2 \mathrm{~s} ; \mathrm{T} 2=1.5^{\circ} \mathrm{C}, 0.8 \pm 0.1 \mathrm{~s} ;$ $\mathrm{T} 2=2.0^{\circ} \mathrm{C}, 1.0 \pm 0.1 \mathrm{~s} ; \mathrm{n}=211$ trials; after capsaicin treatment: $\mathrm{T} 2=0.5^{\circ} \mathrm{C}, 1.6 \pm 0.2 \mathrm{~s} ; \mathrm{T} 2=1.0^{\circ} \mathrm{C}, 0.9 \pm 0.1$ $\mathrm{s} ; \mathrm{T} 2=1.5^{\circ} \mathrm{C}, 0.7 \pm 0.03 \mathrm{~s} ; \mathrm{T} 2=2.0^{\circ} \mathrm{C}, 0.6 \pm 0.05 \mathrm{~s} ; \mathrm{n}=$ 203 trials). We found no changes in light detection latency after capsaicin treatment. However, the occurrence of hold-through trials increased after capsaicin treatment during the light detection trials (before capsaicin, $1 \%$; after capsaicin, $8 \%$ ). Significant reductions of detection latency were observed at the 1.0 and $2.0^{\circ} \mathrm{C} \mathrm{T} 2$ temperatures and a slight decrease was observed at the $1.5^{\circ} \mathrm{C}$ temperature shift, though there was a slight change in detection latency in the $0.5^{\circ} \mathrm{C}$ trials.

\section{Discussion}

We observed that topical administration of capsaicin to the face caused an increase in escape trials, reduction in the number of hold-through trials and an increase in correct trials, and also a significant reduction of detection latency during the cold detection task in monkeys. These data demonstrate that topical administration of capsaicin decreases the cold threshold and hypersensitivity to cold stimulation of the skin. Topical administration of capsaicin to the skin reduces the pain threshold to heat stimulation (6). Capsaicin treatment also enhances extravasation in the capsaicin-treated area, resulting in flare formation in the skin (7).

The capsaicin receptors, TRPV1 and TRPV2, are expressed in $\mathrm{C}$ - and $\mathrm{A} \delta$-fiber terminals, and these fibers are activated by capsaicin administration (8). These terminals showed an increase in excitability following topical application of capsaicin to the skin (7). It has been reported that in humans topical administration of capsaicin to the skin causes hypersensitivity to heat stimulation (3). The reduction of the heat threshold and hyperexcitability of $\mathrm{C}$ - and $\mathrm{A} \delta$-fibers is thought to be involved in the sensitization of peripheral nociceptors. Peripheral sensitization is also thought to be involved in abnormal pain induction (9). These data strongly suggest that topical administration of capsaicin to the skin sensitizes C- and $\mathrm{A} \delta$-fiber terminals.

Recently, it has been reported that TRPV1 and TRPA1 receptors are co-expressed in a large proportion of trigeminal ganglion neurons (4). Also, primary afferent neurons bearing these two receptors are reportedly very common in the trigeminal system compared to the spinal cord. It has also been reported that cold pain threshold is reduced after peripheral inflammation in rats (10). These results strongly suggest that the TRPV1 and TRPA1 receptors are not functionally identical for heat and cold

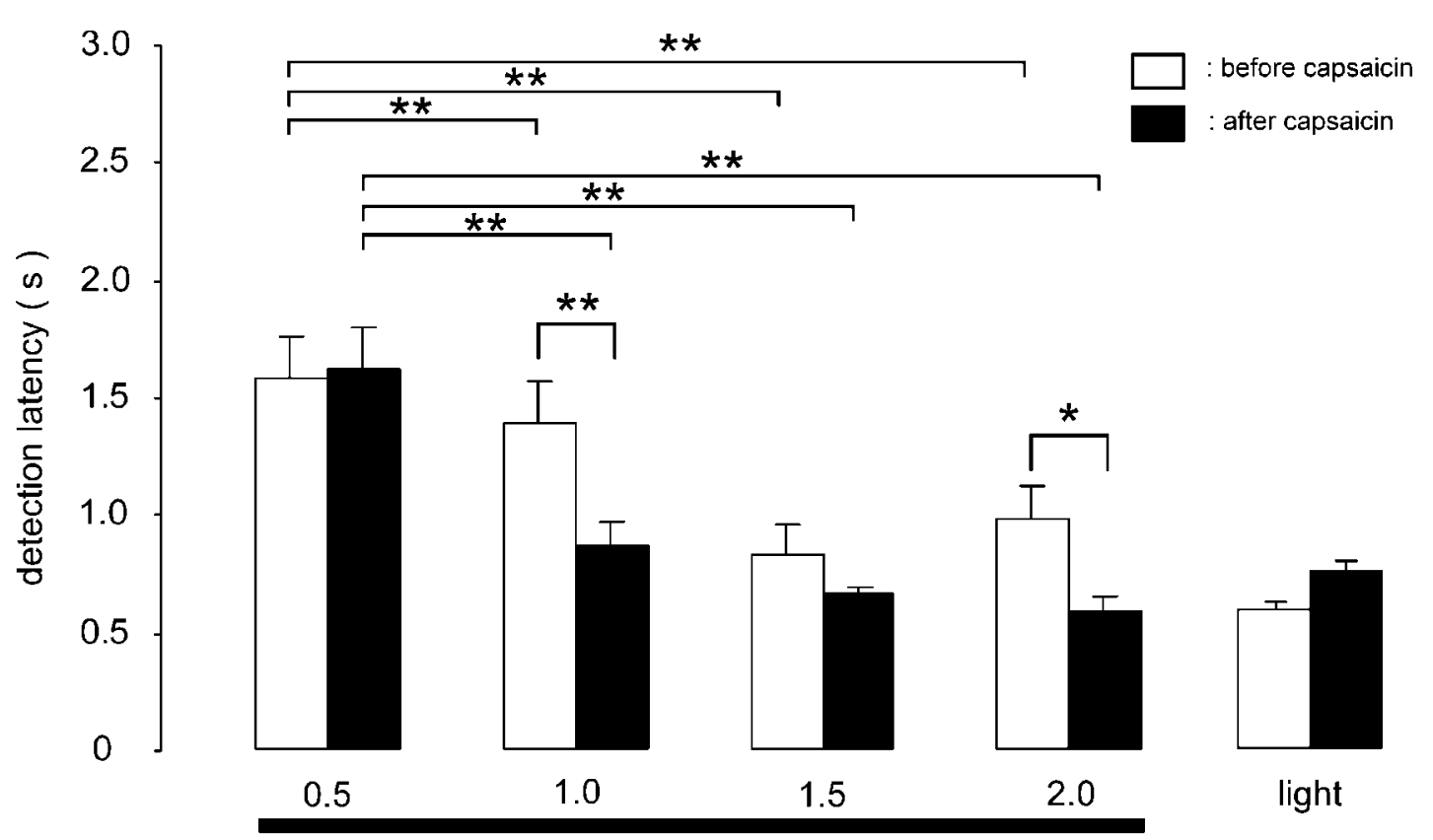

T2 temperature shift $\left({ }^{\circ} \mathrm{C}\right)$

Fig. 3 Detection latencies during the cold and light detection tasks. Hold-through trials were defined when monkeys held the button for over $3 \mathrm{~s}$ after $\mathrm{T} 2$ temperature or V2 light intensity change. $* P<0.05, * * P<0.01$ 
perception.

Together with previous results, the present data suggest that the following two mechanisms may be involved in hyperexcitability to cold stimulation of the skin after capsaicin treatment: 1) $\mathrm{C}$ - and/or $\mathrm{A} \delta$-fiber terminals that express TRPV1 and TRPA1 receptors are directly activated by capsaicin. 2) Peripheral inflammation is induced by capsaicin administration and TRPA1 receptors are activated by a variety of inflammatory "soups."

We also observed an increase in escape and hold-through trials after capsaicin treatment during the light detection task. A number of papers have reported that nociception and other modality sensations such as vision and audition have some interaction with each other (11). It is likely that sensory interactions between cold and vision are involved in modulation of the light detection task.

Nocifensive behavior and noxious reflexes are frequently used in an interchangeable fashion. Our model is a very reliable measure of nocifensive behavior to cold stimulation, rather than a reflex to noxious stimulation. Therefore, the behaving monkey with reversible hypersensitivity induced by capsaicin is a strong tool for studying the neuronal mechanism underlying cold hyperalgesia or cold allodynia.

\section{Acknowledgments}

This study was supported in part by research grants from the Sato and Uemura Funds from Nihon University School of Dentistry, and a grant from the Dental Research Center, Nihon University School of Dentistry; a Nihon University multidisciplinary research grant; a grant from the Ministry of Education, Culture, Sports, Science and Technology for promotion of multi-disciplinary research projects; and a grant from the Ministry of Education, Culture, Sports, Science, and Technology for promotion of multidisciplinary research projects "Brain Mechanisms for Cognition, Memory and Behavior" at Nihon University. We thank Dr. D. A. Thomas (GrammarUSA@starpower.net) for correcting the English of the manuscript.

\section{References}

1. Ringkamp M, Peng YB, Wu G, Hartke TV, Campbell JN, Meyer RA (2001) Capsaicin responses in heat- sensitive and heat-insensitive A-fiber nociceptors. J Neurosci 21, 4460-4468

2. Tominaga M, Tominaga T (2005) Structure and function of TRPV1. Pflugers Arch 451, 143-150

3. Kupers RC, Chen CC, Bushnell MC (1997) A model of transient hyperalgesia in the behaving monkey induced by topical application of capsaicin. Pain 72, 269-275

4. Kobayashi K, Fukuoka T, Obata K, Yamanaka H, Dai Y, Tokunaga A, Noguchi K (2005) Distinct expression of TRPM8, TRPA1, and TRPV1 mRNAs in rat primary afferent neurons with adelta/c-fibers and colocalization with trk receptors. J Comp Neurol 493, 596-606

5. Zimmermann M (1983) Ethical guidelines for investigations of experimental pain in conscious animals. Pain 16, 109-110

6. Davis KD, Kwan CL, Crawley AP, Mikulis DJ (1998) Functional MRI study of thalamic and cortical activations evoked by cutaneous heat, cold, and tactile stimuli. J Neurophysiol 80, 1533-1546

7. Harding LM, Murphy A, Kinnman E, Baranowski AP (2001) Characterization of secondary hyperalgesia produced by topical capsaicin jelly a new experimental tool for pain research. Eur J Pain 5, 363-371

8. Dai Y, Iwata K, Fukuoka T, Kondo E, Tokunaga A, Yamanaka H, Tachibana T, Liu Y, Noguchi K (2002) Phosphorylation of extracellular signal-regulated kinase in primary afferent neurons by noxious stimuli and its involvement in peripheral sensitization. J Neurosci 22, 7737-7745

9. Sessle BJ (2005) Peripheral and central mechanisms of orofacial pain and their clinical correlates. Minerva Anestesiol 71, 117-136

10. Allchone AJ, Broom DC, Woolf CJ (2005) Detection of cold pain, cold allodynia and cold hyperalgesia in freely behaving rats. Mol Pain 1, 36

11. Kenshalo DR Jr, Anton F, Dubner R (1989) The detection and perceived intensity of noxious thermal stimuli in monkey and in human. J Neurophysiol 62, 429-436 